\title{
IL NEO-ISTITUZIONALISMO IN SCIENZA POLITICA: IL CONTRIBUTO DELLA TEORIA DELLA SCELTA RAZIONALE
}

\author{
di Daniela Giannetti
}

\section{Premessa}

Insieme alla ripresa di interesse per il tema dello stato, lo studio delle istituzioni è tornato al centro dell'analisi politologica. $\grave{E}$ opinione condivisa che il rinnovato interesse per le istituzioni rappresenti una reazione alla rivoluzione comportamentista, la quale a sua volta - con l'enfasi sul comportamento osservabile e sui processi informali di potere e influenza - reagiva all'impostazione legalistica e formalistica degli esordi della disciplina ${ }^{1}$. Ciò che, non senza qualche eccesso polemico, è stato definito uno «slittamento paradigmatico» ha coinvolto una varietà di approcci, accomunati dal riconoscimento dei limiti della bebavioral persuasion, e in particolare dei due orientamenti teorici ad essa associati in scienza politica: il pluralismo, con l'accento posto sulla politica come processo e la conseguente messa in ombra degli elementi strutturali, e il funzionalismo, un approccio tendenzialmente più inclusivo ma che in scienza politica si è identificato sostanzialmente con la versione almondiana. Rispetto ai più ambiziosi tentativi teorici del funzionalismo sociologico, essa ha fornito soprattutto uno schema categoriale per l'analisi comparata dei sistemi politici, in cui è attribuito ri-

Ringrazio per $i$ preziosi suggerimenti Nicola Iacobone, Paolo Martelli, Lorenzo Sacconi e gli amici di «Politeia», ai quali una prima versione di questo lavoro è stata presentata nell'ambito del ciclo di seminari 1990-1991. Desidero anche ringraziare Douglas $C$. North e Terry Moe del Dipartimento di Scienza Politica dell'Università di Rochester, N.Y.

${ }^{1}$ Si vedano Riker (1980) e, tra gli altri, Krasner (1984), March e Olsen (1984) ora in March e Olsen (1989), Evans, Rueschemeier e Skocpol (1985). Il saggio di March e Olsen è frequentemente citato come il manifesto del neo-istituzionalismo. In realtà quello che gli autori propongono è una particolarissima versione del revival istituzionale, basata su una generalizzazione del noto modello elaborato per lo studio delle organizzazioni.

RIVISTA ITALIANA DI SCIENZA POLITICA / a. XXIII, n. 1, aprile 1993 
lievo centrale alle funzioni di input o ai processi che vanno dalla società alla politica ${ }^{2}$.

Il tentativo di colmare le lacune del comportamentismo ha generato una vasta letteratura, di carattere teorico ed empirico, $\mathrm{i}$ cui tratti distintivi consistono in una accentuazione degli elementi di regolazione e controllo rispetto all'enfasi pluralista sul processo di allocazione delle risorse, in una ripresa del tema dell'autonomia degli attori istituzionali contrapposta alla concezione dello stato come «arena», nonché nel trattamento esplicito dei vincoli istituzionali come variabile indipendente e nell'analisi dei loro effetti sugli esiti del processo politico. Questa letteratura è caratterizzata da una profonda eterogeneità tanto sotto il profilo teorico - dal momento che in essa confluiscono modelli derivati dalla teoria della scelta razionale, sviluppi dell'analisi marxista e della sociologia storico-comparata di matrice weberiana - quanto sotto il profilo metodologico, dato che approcci che si richiamano all'individualismo metodologico coesistono con spiegazioni di tipo marcatamente strutturale. Tuttavia l'espressione neoistituzionalismo è stata frequentemente usata come formula sintetica per indicare la convergenza di tutte queste differenti prospettive sul terreno dell'analisi delle istituzioni politiche ${ }^{3}$.

Trascurando gli approcci di derivazione sociologica e neomarxista, in questa sede l'attenzione verrà ristretta al neo-istituzionalismo come si configura all'interno della teoria della scelta razionale. Con questa espressione viene comunemente designato nella letteratura un insieme di sviluppi teorici che introduce sensibili elementi di novità sia rispetto alla teoria micro-economica sia rispetto alla prospettiva tradizionale della Public Choice in quanto fortemente influenzata da essa; tanto che secondo alcuni è giustificato parlare di un nuovo paradigma $o$, più esattamente, di un nuovo programma di ricerca ${ }^{4}$. Come è noto, l'ipo-

\footnotetext{
${ }^{2}$ Si veda ad esempio Norlinger (1987). Per una valutazione dell'impatto di questo approccio sulla scienza politica, nel quadro di una più generale analisi delle teorie sistemiche, si vedano Morlino (1989) e Urbani (1989).

${ }^{3}$ Per un panorama dei diversi approcci, si vedano - oltre ai lavori citati alla nota 1 - il dibattito sulle colonne dell'«American Political Science Review» sollevato da Almond (1988). Per un resoconto degli analoghi sviluppi nell'ambito della teoria della scelta razionale che sottolineano il distacco dalla tradizione comportamentista cfr. soprattutto Riker (1980) e Shepsle (1989).

${ }^{4}$ Cfr. ad es. Richter (1984) e Frey (1984). Secondo alcuni il neo-istituzionalismo comporta una modificazione delle «ipotesi periferiche»; secondo altri spinge a una revisione degli assunti comportamentali, ovvero del «nucleo» dell'approccio. Questo punto - sul quale non mi soffermo in questa sede - è discusso da Eggertsson (1990). Precisazioni utili al dibattito sono contenute in Williamson (1990a).
} 
tesi fondamentale del modello dell'attore razionale è che gli individui massimizzino una funzione obiettivo soggetta a vincoli. L'idea portante del neo-istituzionalismo è che le scelte degli attori possano venir comprese adeguatamente solo nel contesto dell'insieme di vincoli e opzioni istituzionalmente generati; da qui l'esplicitazione e l'incorporazione sistematica di tali vincoli, prevalentemente trattati come «esogeni» nei modelli precedenti.

Questo lavoro ha due principali obiettivi. In primo luogo, intendo mostrare come uno dei connotati salienti del programma di ricerca neo-istituzionalista sia costituito da una feconda «ibridazione» tra ambiti disciplinari convenzionalmente separati, tanto da originare un vero e proprio settore di ricerca emergente, che integra su nuove basi l'analisi politica ed economica delle istituzioni (Ordeshook 1990). A tale fine identifico, mostrandone la complementarietà, due importanti direzioni di ricerca all'interno del neo-istituzionalismo: una di esse è ancorata direttamente nella teoria politica positiva, e precisamente in quella letteratura di social choice che ha analizzato estensivamente le procedure di aggregazione delle preferenze individuali, in particolare la regola di maggioranza, a partire dai pionieristici lavori di Black (1958) e di Arrow (1963); l'altra, che ha origine nella teoria economica ed è nota come economia dei costi di transazione, viene fatta risalire ai lavori di Coase (1937), Simon (1957), Alchian e Demsetz (1972) ed è stata sistematizzata da Williamson (1975).

Il secondo obiettivo è quello di mostrare come il programma di ricerca neo-istituzionalista, indirizzandosi a molte questioni di tradizionale interesse politologico, sia sufficientemente maturo per dar vita a un confronto tra scienza politica postcomportamentista e teoria della scelta razionale che resta finora scarsamente sviluppato, anche se non mancano segnali crescenti di interesse reciprocos. A tale fine ne illustro alcune delle potenzialità di sviluppo e di estensione.

Il lavoro è pertanto organizzato come segue. Il primo paragrafo delinea informalmente lo sfondo teorico della social choice che ha condotto all'elaborazione di modelli che incorporano sistematicamente $\mathrm{i}$ fattori istituzionali per spiegare la scelta collet-

5 Si veda ad esempio Almond (1990) e il dibattito suscitato dai saggi raccolti a cura di Monroe (1990a); si veda anche Monroe (1991). L'attenzione di Almond per la teoria della scelta razionale non è nuova: cfr. Almond, Flanagan e Mundt (1973) per un tentativo di utilizzare in maniera combinata questo approccio nell'analisi delle crisi $e$ del mutamento politico. 
tiva, prospettando la direzione di ricerca che scaturisce dall'incontro tra teoria politica positiva ed economia dei costi di transazione per affrontare il problema teorico della genesi e del mutamento istituzionale. Il contesto di riferimento è prevalentemente costituito dalle istituzioni legislative, in particolare il Congresso americano, che sono finora quelle più ampiamente studiate. Nel secondo paragrafo si presentano, sempre in termini non formali, le prime estensioni di questo programma di ricerca a due importanti oggetti di studio: i sistemi parlamentari europei e l'insorgenza delle moderne istituzioni rappresentative. Il paragrafo conclusivo sintetizza alcune implicazioni dell'analisi svolta e discute le più significative obiezioni mosse a questa direzione di indagine nel suo complesso.

\section{Equilibrio e istituzioni: l'approccio della scelta razionale}

Come è noto, uno dei settori analiticamente più sviluppati della teoria politica positiva è costituito dalla teoria della scelta sociale, applicata allo studio delle decisioni di comitato $^{6}$. In modo parallelo allo studio del mercato in economia, l'obiettivo fondamentale di questa analisi è stato la determinazione di equilibri in un contesto istituzionale altamente semplificato come quello costituito dalla regola di maggioranza. Il risultato teorico fondamentale di tre decenni di ricerca in questo settore stabilisce che la regola di maggioranza semplice manca generalmente di proprietà di equilibrio ${ }^{7}$.

Nel contesto unidimensionale l'equilibrio è possibile imponendo condizioni di omogeneità sull'insieme delle preferenze dei votanti. Black ha mostrato che, se tutte le curve di preferenza dei votanti sono a un solo massimo (single-peaked), allora la regola di maggioranza assicura un equilibrio, che coincide con la posizione dell'elettore mediano ${ }^{8}$. Nel contesto multidimensio-

${ }^{6}$ Per un quadro dell'evoluzione teorica di questo settore, allargato anche alla teoria delle elezioni, si vedano Enelow e Hinich $(1984 ; 1990)$. Per una presentazione accessibile dei principali risultati della teoria delle decisioni di comitato vedi l'esaustiva rassegna di Plott (1976) e quella, più aggiornata, di Miller, Grofman e Feld (1989); in lingua italiana si veda Martelli (1983) nel quadro di una più generale analisi.

${ }^{7}$ La possibilità che l'applicazione della regola di maggioranza potesse condurre ad esiti «paradossali» o a cicli fu riconosciuta oltre due secoli fa da Condorcet. Il teorema di impossibilità di Arrow rappresenta una generalizzazione di questo risultato.

${ }^{8} \mathrm{Cfr}$. Black (1948; 1958). Come è noto, questo fondamentale risultato è stato esteso da Downs (1957) alla competizione elettorale. 
nale il potenziale di disequilibrio è sempre presente, a meno di non imporre condizioni estremamente restrittive. Questo risultato è dimostrato in vari teoremi (Plott 1967; McKelvey 1976; Schofield 1978). In particolare il «teorema del caos» dimostrato da McKelvey stabilisce che, date due o più dimensioni e tre o più votanti, per qualsiasi coppia di proposte sottoposte a votazione esiste sempre una sequenza tale per cui ognuna di esse è preferita da una maggioranza alla proposta che la precede nella sequenza. Se esiste un attore dotato in modo esclusivo del potere di strutturazione dell'agenda - per esempio il presidente di una commissione legislativa - questo risultato implica che si dà sempre una sufficiente opportunità di manipolare la sequenza dei voti allo scopo di raggiungere l'esito finale desiderato; se l'agenda è strutturata mediante un processo casuale o aperto, in cui ognuno può proporre una alternativa, allora «il processo può condurre in ogni dove».

In un saggio molto discusso Riker traeva da questi risultati conseguenze epistemologiche più vaste, quali l'impossibilità di costruire una autentica scienza della politica, definita «the dismal science» a causa dell'instabilità della regola di maggioranza e della limitata applicabilità del teorema di Black 9 . Prendere atto della pervasività del disequilibrio ha condotto in realtà all'esplorazione delle sue conseguenze, in particolare all'analisi degli effetti stabilizzanti di strutture e procedure sugli esiti del processo politico.

Il risultato di ciclicità globale individuato al livello di analisi della regola di maggioranza astrattamente considerata si scontra infatti con la stabilità della politica nei contesti reali. Se esiste sempre una maggioranza che può battere una proposta legislativa, come si spiega che nelle istituzioni legislative, ad esempio nel Congresso, si formino coalizioni stabili e che le proposte legislative siano di fatto approvate?

Una risposta alla provocatoria domanda «Wby So Much Stability?» fu avanzata inizialmente da Tullock (1981). Secondo Tullock la ragione va ricercata nelle pratiche di contrattazione e scambio dei voti (logrolling). Poiché l'intensità delle preferenze

9 Cfr. Riker (1980), che riprende la nota espressione adoperata da Carlyle a proposito dell'economia. Il dibattito teorico sollevato da questo saggio è raccolto nel volume antologico curato da Ordeshook e Shepsle (1981). Successivamente Riker ha almeno in parte modificato le posizioni scettiche espresse in questo saggio: si veda ad es. Riker (1990). 
sulle differenti questioni non è uniformemente distribuita tra $\mathrm{i}$ legislatori, l'approvazione di una proposta legislativa passa attraverso un accordo informale che conduce a uno scambio di voti da cui tutti traggono vantaggio. Benché questa risposta abbia il merito di spostare il fuoco dall'aggregazione delle preferenze allo scambio, non risolve il problema dell'instabilità per $\mathrm{i}$ seguenti motivi: a) il logrolling non sfugge all'instabilità dovuta alla distorsione strategica delle preferenze. Un legislatore che beneficia della proposta $X$ potrebbe opporsi ad essa e assicurarsi il sostegno su un'altra in cambio del suo voto su $\mathrm{X}$. In questo modo otterrebbe il risultato preferito sia in merito ad $\mathrm{X}$ sia in merito ad altre proposte. Se anche altri mettono in moto un processo di bluffing, può essere impossibile determinare l'esito finale; b) se gli agenti dello scambio sono coinvolti in accordi informali e le proposte sono votate una alla volta c'è un incentivo al rinnego, cioè un problema di esecuzione (enforcement) degli accordi (Mueller 1989, 91-94).

La risposta neo-istituzionalista è che gli esiti del processo legislativo siano determinati non solo dalle preferenze degli attori coinvolti, ma anche dai vincoli strutturali e procedurali che trasformano la regola di maggioranza in un assetto istituzionale più complesso. Se il logrolling non sfugge all'instabilità, occorre guardare non allo scambio in quanto tale, ma alle regole che restringono le possibilità coalizionali e di scambio, cioè che rendono eseguibili solo alcuni tipi di scambi (Shepsle e Weingast 1981). L'obiettivo di incorporare in modo non idiosincratico tali vincoli nel trattamento formale sta alla base dell'elaborazione di una ormai vasta classe di modelli, noti come modelli di equilibrio indotto dalla struttura (Structure Induced Equilibrium o $S I E)^{10}$. Il fuoco è su due elementi delle istituzioni: quello strutturale, cioè i meccanismi di divisione e specializzazione del lavoro, e quello procedurale (quali alternative vengono considerate, in quale ordine ecc.).

I modelli spaziali tradizionali assumono tipicamente un insieme anonimo di votanti, mentre la differenziazione - in dipartimenti, commissioni e così via - costituisce un fattore centrale di molti contesti decisionali. Il corpo legislativo viene pertanto trattato come un insieme di sub-unità distinte che, nel contesto

\footnotetext{
${ }^{10}$ Questo risultato è dimostrato per la prima volta in Shepsle (1979). Una presentazione non tecnica si trova in Shepsle (1986) e Shepsle (1989).
} 
legislativo, corrispondono al sistema delle commissioni. Parallelamente, anziché postulare un insieme indifferenziato di alternative, lo spazio politico è suddiviso in specifiche giurisdizioni, corrispondenti ad altrettante dimensioni dello spazio. Nel contesto americano, un referente legislativo è ad esempio la Regola $\mathrm{X}$ della Camera, che elenca le commissioni permanenti e le specifiche aree di competenza loro assegnate. L'assunto è che esista una relazione uno-a-uno tra commissioni, giurisdizioni e dimensioni dello spazio. Ciascuna sub-unità possiede specifici diritti in relazione a ciascuna giurisdizione, in particolare il monopolio dell'iniziativa politica in quell'area di competenza e il diritto di veto. Solo la commissione può proporre alterazioni dello status quo nella sua giurisdizione, altrimenti può decidere di «tenere le porte chiuse». Nel modello più semplice, il processo decisionale è articolato in due stadi. Se la commissione avanza una proposta, il corpo legislativo nel suo complesso approva, rifiuta o emenda attraverso la votazione a maggioranza. Le regole procedurali che governano il processo di emendamento sono di fondamentale importanza, in quanto corrispondono a restrizioni significative sull'ambito delle comparazioni possibili. L'incorporazione dell'elemento procedurale segna l'altro punto di distacco dai modelli spaziali tradizionali. Nel contesto legislativo, un concreto referente è ad esempio la germaneness rule, cioè una regola che restringe gli emendamenti possibili a quelli pertinenti a una singola giurisdizione.

Questo insieme di elementi - articolazione in commissioni, differenziazione giurisdizionale, potere di agenda delle commissioni sulle rispettive giurisdizioni, regole di emendamento è sufficiente per modellare un assetto istituzionale ancora «stilizzato», ma assai meno «spartano» di quello descritto da una semplice regola di aggregazione delle preferenze. La conseguenza teorica di questi assunti è che la votazione delle proposte su una dimensione alla volta - se $i$ votanti votano «sinceramente» - conduce tipicamente a scelte mediane su ciascuna dimensione $^{11}$.

La derivazione di questo risultato ha due implicazioni. Sotto il profilo teorico, rappresenta una soluzione al problema dell'instabilità, come formulato dai padri fondatori della teoria del-

"Sulla distinzione tra voto «sincero», o in accordo con le proprie autentiche preferenze, e voto «sofisticato» o strategico cfr. Farquharson (1969). 
la scelta collettiva. In uno spazio multidimensionale l'equilibrio è «indotto» dall'esistenza di determinati fattori strutturali e procedurali, che - agendo come binding constraints sul comportamento degli attori - rendono possibile la scelta legislativa. Le tradizionali conclusioni della letteratura di social choice risultano pertanto sostanzialmente modificate: in particolare, mentre mantengono la loro validità nell'ambito della regola di maggioranza pura, non si applicano ai più complessi contesti reali. Il riconoscimento dell'importanza del ruolo dei fattori istituzionali nella determinazione del comportamento legislativo e, in ultima istanza, della public policy modifica l'agenda della ricerca indirizzando l'attenzione verso l'analisi delle proprietà di tali assetti.

La seconda implicazione scaturisce dalle possibili interpretazioni di questo risultato. In questo contesto un equilibrio è semplicemente un esito stabile, che può essere collocato lontano dall'intersezione delle mediane su ogni dimensione di tutta la legislatura. È stato possibile allora interpretare questo risultato come una conferma teorica dell'ipotesi secondo cui le commissioni eserciterebbero una «sproporzionata» influenza sulla scelta legislativa, a spese della maggioranza. A partire da questa interpretazione sono stati elaborati numerosi modelli volti ad affrontare la questione del «chi ottiene che cosa a spese di chi» in presenza di differenti assetti istituzionali ${ }^{12}$. Questa seconda generazione di modelli si caratterizza per due aspetti rilevanti rispetto al più semplice modello di partenza: a) modificazione degli assunti comportamentali, con l'introduzione di diversi gradi di anticipazione razionale; b) esplorazione di più complessi assetti istituzionali (regole procedurali complesse, giurisdizioni multidimensionali, sequenze a più stadi in cui è articolato il processo decisionale). Un esempio è il modello elaborato da Shepsle e Weingast (1987) allo scopo di esplorare le fondamenta istituzionali del potere delle commissioni. La loro analisi conduce alla conclusione secondo cui le commissioni beneficiano in modo «sproporzionato» dalla scelta legislativa, cioè riescono a far approvare proposte legislative contrarie alla maggioranza, solo in presenza di certe protezioni procedurali, ad esempio un veto ex post, che oltre ad agire come strumento offensivo consente anche di condizionare il comportamento della maggioranza parlamentare. Indipendentemente dall'adeguatezza delle pre-

12 Per una esauriente rassegna critica si veda Krehbiel (1988). 
visioni empiriche da essi derivate, la seconda e più generale implicazione di questi risultati consiste allora nell'accentuazione delle potenzialità positive della teoria della scelta sociale, dal momento che $\mathrm{i}$ modelli di equilibrio indotto dalla struttura si indirizzano all'analisi della natura e delle conseguenze di differenti assetti istituzionali. Detto altrimenti, essi sono concepiti come strumenti di analisi comparata delle istituzioni e, in particolare, di quelle che possono essere definite le loro proprietà distributive.

Nei termini di Shepsle (1986), gli obiettivi del neo-istituzionalismo sono tuttavia più ambiziosi. Comprendere gli effetti delle istituzioni sugli esiti del processo politico, o formulare una teoria dell'«equilibrio istituzionale», rappresenta un passo importante, ma preliminare. Il secondo obiettivo è spiegare genesi e mutamento istituzionale, cioè formulare una teoria delle «istituzioni in equilibrio». «Endogenizzare» le istituzioni, cioè spiegare selezione e mutamento di differenti assetti istituzionali a partire dalle scelte individuali di attori razionali, appare tanto più decisivo per una prospettiva che si fonda sull'individualismo metodologico. Questo compito non è soddisfatto dai precedenti modelli, dal momento che essi non affrontano la questione della scelta dei vincoli, trasferendo il problema dell'instabilità dal dominio delle policies a quello delle «regole del gioco» ${ }^{13}$.

Come è noto, entro l'approccio della scelta razionale sono stati perseguiti diversi tentativi di render conto dell'insorgenza delle istituzioni, alcuni dei quali - concepiti con esplicito orientamento normativo - sono talora offerti più come una metafora di quali istituzioni sarebbero selezionate da attori razionali che come una spiegazione vera e propria (Rawls 1971; Buchanan e Tullock 1962; Harsanyi 1977). Una distinzione importante che è emersa nella letteratura di teoria dei giochi è quella tra istituzioni che sorgono in risposta a problemi di coordinazione e selezione di istituzioni cooperative, cioè «soluzioni negoziate al problema dell'azione collettiva», che implicano differenti surplus per gli attori e sono vulnerabili al free-riding. Mentre nel primo caso la genesi istituzionale appare suscettibile di una

${ }^{13} \mathrm{La}$ distinzione tra scelta di corsi d'azione alternativi entro un assetto istituzionale dato e scelta tra sistemi di regole alternative - o stadio parlamentare e costituzionale del decision-making - è stata sottolineata da Buchanan a partire dalla fondamentale opera scritta in collaborazione con Tullock. Cfr. Buchanan e Tullock (1962). 
spiegazione «endogena», ovvero in termini di equilibri emergenti da una molteplicità di azioni complementari e capaci di perpetuarsi in quanto Pareto-efficienti ${ }^{14}$, il secondo problema esemplificato da situazioni di tipo Dilemma del prigioniero presenta, come è noto, assai maggiori difficoltà per una spiegazione in termini di razionalità individuale. In questo contesto il problema non consiste soltanto nel fornire una spiegazione dell'insorgenza della cooperazione, data la struttura degli incentivi, ma nel rendere conto dei meccanismi centralizzati di enforcement. Gran parte dell'analisi condotta entro la teoria dei giochi ha esplorato le condizioni alle quali emerge e si sostiene la cooperazione in assenza di autorità centralizzata. L'interazione ripetuta può creare le condizioni alle quali i prospetti di vincite future inibiscono il comportamento non cooperativo in situazioni del tipo Dilemma del prigioniero. Un altro meccanismo che consente l'instaurarsi della cooperazione è la reputazione ${ }^{15}$. Pur offrendo un contributo imprescindibile all'analisi del problema, $\mathrm{i}$ resoconti in termini di teoria dei giochi restano controversi se assunti come base di una spiegazione comprensiva della genesi istituzionale ${ }^{16}$.

\section{L'economia dei costi di transazione}

La prospettiva che si è recentemente imposta come particolarmente fruttuosa allo scopo di affrontare il problema della genesi delle istituzioni è l'economia dei costi di transazione ${ }^{17}$. Essa

14 Un equilibrio si definisce Pareto-efficiente quando non potrebbe attuarsi alcuna riallocazione o scambio capace di migliorare ulteriormente la situazione di tutti gli attori coinvolti. L'argomento della «mano invisibile», risalente a Mandeville e Smith e ripreso in questo secolo soprattutto da Hayek, è stato riproposto nell'ambito della moderna teoria dei giochi: cfr. ad esempio Schotter (1981). Sull'insorgenza di convenzioni i volumi di riferimento sono Schelling (1960) e Lewis (1962); per una ricostruzione nei termini della teoria dei giochi dei differenti contesti che motivano l'insorgenza di istituzioni si veda Ulmann-Margalit (1977).

is La differenza tra giochi iterati e one-shot games per quello che riguarda le differenti prospettive di cooperazione è già sottolineata in Luce e Raiffa (1957); cfr. Taylor (1976) e Axelrod (1984) per uno sviluppo di questo argomento. Per l'incorporazione della reputazione nel trattamento formale cfr. Kreps e Wilson (1982).

16 Il risultato teorico fondamentale che prova definitivamente la possibilità di insorgenza «endogena» della cooperazione ma anche la molteplicità degli equilibri nei giochi iterati è dimostrato da Fudenberg e Maskin (1986). Per le critiche mosse a questo approccio si vedano Hetcher (1990) e Bendor e Mookherjee (1987).

${ }_{17} \mathrm{Si}$ veda, in particolare, Williamson $(1975 ; 1985)$. Una presentazione sintetica è 
trae origine dall'insoddisfazione per la teoria microeconomica neoclassica, focalizzata quasi esclusivamente sul mercato e sull'impresa come concepiti all'interno di modelli altamente astratti e semplificati. Pur consentendo di derivare potenti ipotesi a livello di proprietà aggregate dei sistemi economici sulla base di un ristretto insieme di assunti di razionalità relativi agli attori individuali, la teoria microeconomica neoclassica ha mostrato non solo limiti esplicativi - ad esempio in relazione alle diverse modalità di organizzazione del processo produttivo - ma veri e propri problemi di coerenza interna ${ }^{18}$.

Il nucleo principale dell'economia dei costi di transazione è l'idea che gli scambi - che rappresentano la fondamentale unità d'analisi - non siano privi di costi, a differenza di quanto postulato nei modelli neoclassici, e che le istituzioni economiche contano (do matter) precisamente per questo. All'interno di questa prospettiva resta centrale l'orientamento alla massimizzazione che caratterizza la teoria microeconomica e in generale il modello dell'attore razionale. Tuttavia, l'esplicito riconoscimento della razionalità limitata $\mathrm{da}$ un lato, e l'assunto che il perseguimento del self-interest si connoti frequentemente in modo «opportunistico» dall'altro, dirigono l'attenzione verso modalità di organizzazione delle transazioni alternative allo scambio di mercato. Solo assumendo che gli attori siano limitatamente razionali e operino in un ambiente mutevole e strategico $i$ costi di transazione diventano salienti, e così le istituzioni ${ }^{19}$.

Due tipi di costi sono particolarmente importanti: a) costi di misurazione; b) costi di esecuzione (enforcement) degli accordi. Nel contesto dello scambio di mercato il primo problema è relativo ai costi di informazione per misurare gli attributi dei beni e servizi che vengono scambiati. La letteratura ha analizzato come

stata proposta dallo stesso Williamson in numerosi articoli; cfr. ad es. Williamson (1990b). Tra le numerose rassegne, rimando al già citato volume di Eggertsson (1990).

${ }^{18}$ L'insorgenza stessa dell'impresa resta inspiegata in questa prospettiva, dal momento che in base agli assunti tutti gli scambi dovrebbero essere organizzati secondo la modalità di contrattazione atomistica che è tipica del mercato. Per una discussione di questi aspetti si vedano ad es. Barney e Ouchi (1986) e Moe (1984).

19 Williamson (1985) propone una esplicita revisione degli assunti comportamentali su cui si fonda il modello dell'attore neo-classico. Per quello che riguarda gli assunti cognitivi, il riferimento è alla razionalità limitata di Simon, di cui viene tuttavia rifiutato lo «psicologismo» dei livelli di aspirazione. Per quello che riguarda gli assunti motivazionali, Williamson distingue vari livelli di ricerca del self-interest: l'opportunismo, che implica calcolati tentativi di manipolazione, ne rappresenta la forma più estrema. 
questi costi influenzino lo scambio sia ex ante sia ex post ${ }^{20}$. Il secondo tipo di costi riguarda i costi di assicurare l'esecuzione del contratto. La letteratura sui costi di transazione sottolinea il fatto che mentre le parti possono avere forti incentivi a stipulare un accordo, i loro incentivi a rispettarlo dopo che esso è stato stipulato possono cambiare. Il rispetto dell'accordo è reso problematico da vari fattori. Un tema centrale dell'analisi di Williamson è quello dell'incompletezza costitutiva dei contratti, cioè l'impossibilità di specificare in modo completo ex ante - data la razionalità limitata - le possibili contingenze future. Un'altra variabile è la specificità degli investimenti che caratterizzano la transazione. Sono specifici quegli investimenti effettuati a sostegno di una transazione particolare, il cui valore viene a dipendere dalle prospettive di continuità della relazione.

Centrale entro questo approccio è l'idea che, quando i problemi ex post sono anticipati ex ante, gli attori tenteranno di alterare gli incentivi, creando istituzioni che inibiscono l'opportunismo ex post, tipicamente attraverso meccanismi di integrazione verticale o altre forme di governo delle transazioni diverse dallo scambio di mercato. In sintesi, nucleo di questo framework è l'ipotesi che forme alternative di organizzazione economica, o strutture di governo delle transazioni, si sostituiscano allo scambio di mercato per minimizzare $i$ costi di transazione.

Mentre la generalizzazione del paradigma contrattuale ha preso diverse strade ${ }^{21}$, assume particolare importanza per i nostri scopi l'estensione dell'analisi in termini di costi di transazione alle istituzioni legislative. Nel contesto legislativo, il problema è quello di rendere esecutivi gli accordi tra una molteplicità di attori che rappresentano differenti interessi, per esempio i membri del Congresso. L'idea di fondo è che l'assetto istituzionale - strutture e procedure - mediante il quale è organizzato il

20 Il caso illustrativo del primo problema, trattato in un classico articolo di Akerlof (1970), è quello della vendita di auto usate, di cui solo il venditore conosce pregi e difetti, con i conseguenti problemi di uselezione contrariar. Brand names e garanzie del prodotto sorgono per contrastare questa situazione. L'altro caso, noto come «rischio morales, si riferisce ai problemi di controllo della prestazione dopo che il contratto è stato stipulato.

${ }^{21}$ Si veda ad esempio Milgrom e Roberts (1988) per una estensione in termini di costi di governo o dell'autorità centralizzata. Per interessanti applicazioni all'analisi delle organizzazioni pubbliche attraverso il modello principale-agente si vedano Tirole (1986) e Moe (1990a). 
Congresso americano sia tale da mitigare i costi di transazione che si presentano nel contesto dello scambio legislativo.

Un modello compiuto è elaborato da Weingast e Marshall (1988). Si assume che gli attori perseguano la rielezione procurando benefici ai loro rappresentati e traggano benefici dallo scambio a causa della diversa intensità delle preferenze su una molteplicità di questioni. Questa diversità emerge con chiarezza nel sistema legislativo americano, in cui i rappresentanti politici sono legati a specifiche constituencies geografiche.

Le domande a cui il modello si indirizza sono le seguenti: come sono catturati i «guadagni dallo scambio»? come sono fatti rispettare gli accordi? Lo scambio è vulnerabile a problemi di enforcement, trascurati nei modelli di «mercato dei voti» che assumono l'informazione completa e sopprimono la dimensione temporale. Una varietà di scambi poggia su impegni relativi a contingenze future, cioè a promesse di voti.

Weingast e Marshall identificano due casi in cui il problema dei costi di esecuzione dello scambio è rilevante: scambi non contemporanei e flussi non simultanei di benefici. Il primo caso sorge quando i possibili scambi riguardano proposte che non vengono sottoposte a votazione contemporaneamente, con uno o più parlamentari che promettono appoggio sulla proposta che sta per essere votata in cambio di un voto su una proposta che verrà votata in futuro. L'inosservabilità e l'impossibilità di specificare adeguatamente le contingenze future - per esempio imprevisti mutamenti elettorali - limitano fortemente le possibilità di scambio. Il secondo caso è, ad esempio, quello di un gruppo di legislatori che ricerchi il sostegno su un determinato programma di spesa nel proprio collegio elettorale, in cambio del sostegno offerto a un altro gruppo che persegue l'approvazione di un'altra proposta a beneficio dei propri elettori. L'accordo è vulnerabile al rinnego ex post. II primo gruppo, dopo l'approvazione della proposta legislativa che lo favorisce, potrebbe formare una coalizione con un altro gruppo per revocare la proposta che beneficia il partner. Anche se l'interazione ripetuta e la reputazione sono importanti per dar conto dell'emergere di molte pratiche informali, non assicurano il self-enforcement in queste situazioni.

$\grave{E}$ ai problemi che sorgono nel contesto dello scambio legislativo che occorre guardare per comprendere la genesi dei vari meccanismi procedurali operanti nel Congresso americano. Essi corrispondono, ad esempio, alla regola di non votare una alla 
volta le varie proposte, ma di votare un pacchetto complessivo di proposte legislative («omnibus bill»), prevenendo così la possibilità di rinnego. Tuttavia il problema si ripresenta se questi scambi devono avvenire ex novo in varie occasioni. Uno strumento più efficiente per proteggersi dall'opportunismo è l'organizzazione del Congresso in base al decentramento in un sistema di comitati, ciascuno dei quali dotato del monopolio di iniziativa politica nell'area di propria competenza e del controllo sull'ordine del giorno. Con il decentramento imposto dal sistema dei comitati il problema del rinnego ex post è fortemente ridimensionato dal fatto che ciascuna commissione possiede un diritto di veto rispetto a iniziative legislative nell'ambito delle materie di sua competenza.

La conclusione cui pervengono Weingast e Marshall è che «invece di scambiare voti, i parlamentari scambino diritti speciali che attribuiscono ai loro possessori capacità addizionali di influenza su ambiti di competenza ben definiti. Questa influenza deriva dai diritti di proprietà stabiliti sui meccanismi di agenda, cioè sullo strumento mediante il quale le alternative sono sottoposte al voto. Questa speciale influenza su particolari settori di competenza istituzionalizza uno specifico modello di scambi. [...] Poiché lo scambio è istituzionalizzato, non necessita di essere rinegoziato ad ogni sessione legislativa ed è soggetto a minori problemi di esecuzione ex postı (Weingast e Marshall 1988, 198).

Mentre la forma specifica dei problemi di transazione che si incontrano nelle legislature differisce rispetto a quelli che si incontrano nei mercati, vale la lezione generale della Nuova Economia Istituzionale. Le istituzioni sorgono per abbassare i costi di transazione, cioè possiedono aspetti di salvaguardia contro il rinnego, l'opportunismo e altre circostanze avverse. Esse possono venir interpretate come accordi ex ante, l'obiettivo dei quali è rendere possibile la cooperazione. Nel contesto dello scambio di mercato, i problemi di incentivo sorgono dalla specificità degli investimenti. Nel contesto politico i problemi di incentivo sorgono perché lo scambio dei voti, che comporta l'assunzione di impegni futuri, implica la possibilità di rinnego. Ciò motiva il disegno di istituzioni volte a conferire stabilità agli accordi, in modo analogo al sorgere di meccanismi di integrazione verticale nel contesto dello scambio di mercato. Questo modello illustra significativamente la complementarietà tra le due direzioni di ricerca, gettando un ponte tra teoria della scelta collettiva ed economia dei costi di transazione. I problemi di esecuzione dello 
scambio hanno infatti un'origine fondamentalmente politica, in quanto sono radicati nell'instabilità della regola di maggioranza. E questo l'anello di congiunzione tra la direzione di ricerca che esplora le implicazioni del disequilibrio e l'economia dei costi di transazione, ed è a partire da qui che l'analisi politica può spingere verso estensioni non ovvie del paradigma contrattuale.

\section{Estensioni del programma di ricerca neo-istituzionalista}

Il progetto di estendere il programma di ricerca neo-istituzionalista sia in senso che potremmo definire «orizzontale», cioè all'analisi di altri assetti istituzionali democratici, sia in senso «longitudinale» o diacronico, cioè all'analisi della genesi e del mutamento storico di assetti istituzionali pre-democratici, è stato appena intrapreso. Tuttavia l'importanza di questa estensione appare evidente, se l'obiettivo è quello di gettare le basi di una teoria delle istituzioni dotata di sufficiente generalità, verificando il potenziale degli strumenti analitici in differenti contesti. In questo paragrafo presento alcuni risultati nell'ambito di entrambe le direzioni di ricerca.

Anche i modelli elaborati per studiare i sistemi parlamentari combinano i risultati dell'analisi spaziale condotta nell'ambito della social choice con i concetti dell'economia dei costi di transazione, applicandoli allo studio del processo di formazione di governi di coalizione (Shepsle e Laver 1990a; 1990b). Punto di partenza è che in questo contesto gli attori rilevanti siano i partiti. L'assunto motivazionale è che ciascuno degli attori sia interessato a che il programma politico del governo rifletta il più possibile le proprie preferenze sia per ragioni intrinseche sia per ragioni elettorali.

Rispetto alla teoria delle coalizioni, i modelli neo-istituzionalisti introducono due innovazioni chiave. La prima è la distinzione - finora trascurata al più astratto livello di analisi tipico del trattamento formale - tra coalizioni finalizzate all'approvazione di una proposta legislativa, quali quelle che si formano ad esempio nel Congresso americano, e coalizioni governative, caratterizzate da un accordo su un ampio ventaglio di questioni e dal controllo esecutivo sulle dimensioni politiche salienti. La seconda innovazione riguarda la credibilità delle proposte di formazione di un nuovo governo. La formazione di un governo consiste in un accordo tra i membri della coalizione in ordine al 
perseguimento di un determinato programma politico. Nei sistemi parlamentari un elemento caratteristico dei governi è il meccanismo di assegnazione dei ministeri, che conferisce ai loro titolari potere di agenda ovvero il diritto di iniziativa politica in una determinata area di competenza. La negoziazione che conduce alla formazione del governo implica promesse reciproche su quali politiche verranno implementate. Tuttavia, non tutte le promesse sono credibili: ad esempio, se un partito ambientalista è membro della coalizione e controlla il ministero dell'ambiente, si può ipotizzare che non verranno implementate politiche contrarie agli interessi dei suoi rappresentati, previste o meno dall'accordo di coalizione.

Pertanto si assume che siano ritenuti credibili dagli attori solo quegli accordi politici che risultano effettivamente implementabili mediante l'attribuzione di specifiche responsabilità ministeriali. Questo elemento ha un considerevole impatto sul modo in cui viene modellato il processo di formazione di coalizioni. L'esistenza di un numero finito di ministeri comporta la restrizione delle scelte politiche implementabili da una coalizione, e quindi la riduzione del numero delle coalizioni potenziali. Secondo Shepsle i modelli formali delle coalizioni hanno finora trascurato il problema della credibilità degli accordi e quello della necessità di strumenti di enforcement. È questo il motivo che spiega la loro indeterminazione, parallela al problema dell'instabilità in ambito legislativo.

Una volta che una coalizione si è formata, l'accordo di governo è esposto a due tipi di problemi di enforcement. In primo luogo il governo potrebbe perseguire su una determinata questione una politica diversa da quella concordata con un membro della coalizione. In secondo luogo, alcuni membri della coalizione potrebbero avere incentivi a rinnegare gli accordi opponendosi alla realizzazione di alcune delle politiche promesse ad altri.

L'idea è quella di trattare la divisione dei ministeri in modo analogo al sistema delle commissioni nel Congresso. Il meccanismo di assegnazione dei ministeri mitiga il primo problema. La delega del potere di iniziativa legislativa attraverso i ministeri limita la capacità dei leader governativi di perseguire politiche diverse da quelle promesse perché il ministero rilevante possiede il diritto di iniziare la legislazione in quell'area e monopolizza l'informazione. Quindi i progetti di altri membri della coalizione possono essere bloccati da colui che occupa il ministero rilevante. 
Esiste tuttavia un secondo problema, che è quello di ottenere la cooperazione degli altri membri della coalizione per far approvare una iniziativa legislativa. Un elemento saliente di quasi tutti i sistemi parlamentari è che il governo può essere battuto da un voto di fiducia. $\grave{E}$ sempre possibile che parte della coalizione governativa decida di rinnegare l'accordo con uno dei membri evitando di perseguire una determinata politica promessa a quest'ultimo. La capacità di rovesciare il governo attraverso il meccanismo del voto di fiducia impone tuttavia un vincolo sull'incentivo al rinnego, poiché esiste una minaccia che il membro della coalizione possa unirsi all'opposizione parlamentare nel votare la sfiducia al governo. In modo analogo alla proposta legislativa omnibus nel contesto americano, il voto di fiducia può essere interpretato come uno strumento di esecuzione dell'accordo di governo, volto a prevenire tentativi unilaterali di alterarne i termini ex post.

Mentre le conseguenze più immediate di questa analisi riguardano le previsioni relative al processo coalizionale, che risultano sostanzialmente diverse tanto rispetto ai modelli officeseeking quanto ai modelli policy-seeking ${ }^{22}$, essa contiene anche implicazioni più generali per la teoria politica positiva. L'incorporazione dei fattori istituzionali nell'analisi del processo di formazione delle coalizioni costituisce infatti un primo passo verso l'unificazione di settori di ricerca finora distinti, nel tentativo di pervenire a una sempre più accurata comprensione della dinamica coalizionale e della stabilità governativa.

Rispetto alla teoria delle coalizioni, mentre conduce a una riconsiderazione del size principle come caso particolare, valido solo in un contesto atomistico e institution-free, offre un modo di spiegare razionalmente le presunte «deviazioni» da esso, quali i governi minoritari ${ }^{23}$. La distinzione tra coalizione legislativa e coalizione di governo è essenziale. Un governo minoritario,

${ }^{2}$ Questa distinzione si riferisce alle assunzioni motivazionali che sono state impiegate nella costruzione di modelli teorici del processo coalizionale (Laver e Schofield 1990). Nei modelli spaziali multidimensionali elaborati da Shepsle e Laver gli attori sono interessati alle policies che verranno implementate dal governo che si formerà, $e$ ciascuno di essi valuta la combinazione di scelte politiche risultante dalla formazione di un governo in termini di distanza euclidea dalla sua posizione ideale.

${ }^{23}$ Cfr. Shepsle e Laver (1990c). Il size principle, o la previsione che si formeranno coalizioni minime vincenti, rappresenta il risultato teorico fondamentale su cui si è edificata la teoria delle coalizioni (Riker 1962). Per una spiegazione alternativa, ma non incompatibile, dei governi minoritari nei termini del modello dell'attore razionale cfr. Strom (1990). 
definito come una assegnazione di ministeri a partiti che non controllano una maggioranza legislativa, può rappresentare un equilibrio in talune circostanze, ovvero laddove non esistano proposte alternative credibili. Analogamente, membri non necessari per la maggioranza legislativa possono risultare essenziali per la credibilità di una proposta di governo, come accade nel caso dei surplus majority governments. Per quanto riguarda i più generali obiettivi di edificazione teorica, mentre l'incorporazione di ulteriori vincoli istituzionali - di natura procedurale o legati a specifiche posizioni strutturali, quali il ruolo di Primo Ministro - offre la promessa di modelli sempre più complessi e realistici, il trattamento sistematico di fattori quali il meccanismo di assegnazione dei ministeri e il voto di fiducia, concepiti come «soluzione» a problemi di enforcement degli accordi, mostra sufficientemente le potenzialità di estensione del paradigma contrattuale e offre un nuovo terreno per la verifica delle previsioni da esso derivate ${ }^{24}$.

Veniamo ora all'analisi degli assetti istituzionali pre-democratici. Benché essa rappresenti una sfida di elevata complessità, data l'attenzione quasi esclusiva per le democrazie contemporanee che ha finora caratterizzato la teoria della scelta razionale in scienza politica, i risultati che provengono dall'incontro tra neoistituzionalismo e ricerca storico-comparata non rappresentano soltanto un brillante esempio delle potenzialità analitiche dell'approccio, ma anche un passo ulteriore verso l'integrazione teorica tra economia e politica. L'elaborazione del più compiuto tentativo in questa direzione muove infatti dal tentativo di affrontare il classico problema delle variazioni nella crescita economica (North 1981; 1990).

L'ipotesi centrale di North è che la crescita economica sia determinata non solo da fattori tecnologici e demografici, ma soprattutto dai fattori istituzionali, in particolare dalla struttura dei diritti di proprietà, che definiscono il possesso e l'uso delle risorse economiche, così come sono stabiliti e fatti rispettare dall'autorità politica. Questi fattori sono trascurati entro la prospettiva classica, che procede in base all'assunto secondo cui $\mathrm{i}$ diritti di proprietà sono sempre specificati e fatti rispettare senza costi. Una teoria della crescita economica richiede pertanto

${ }^{24}$ Cfr. Laver e Shepsle (1990a) circa la possibilità di incorporare ulteriori vincoli nel trattamento formale e per una prima applicazione all'analisi del processo coalizionale in Islanda e in Irlanda. 
una teoria delle istituzioni politiche, che estenda allo scambio politico i concetti e gli strumenti dell'economia dei costi di transazione. La generalizzazione della prospettiva contrattuale allo studio delle istituzioni politiche viene sviluppata a partire $\mathrm{da}$ un modello semplificato - in cui agiscono un governante e i suoi constituents - che può essere interpretato come l'embrione di una teoria della formazione dello stato moderno ${ }^{25}$. Il governante offre a differenti gruppi sociali beni collettivi quali protezione e giustizia, o almeno riduzione del disordine interno e salvaguardia di alcuni diritti di proprietà, in cambio di ricavi fiscali. L'ipotesi è che il governante tenterà di massimizzare i ricavi fiscali per sé, agendo come «monopolista discriminante» nei confronti dei vari gruppi di sudditi. Economie di scala nella produzione di beni pubblici quali sicurezza e ordine interno accrescono i benefici totali, ma la distribuzione interna di tali benefici varia a seconda del potere di contrattazione relativo, che dipende sia dal potenziale di violenza del governante sia dai costi opportunità dei sudditi (su cui influisce la possibilità di exit). Il modello viene successivamente reso più complesso introducendo, da un lato, un corpo rappresentativo - come i parlamenti che sorgono tipicamente agli albori dell'Europa moderna - che riflette gli interessi dei rappresentati e il loro ruolo nel processo di contrattazione con i governanti e, dall'altro, una struttura gerarchica di «agenti», ovvero una burocrazia, a cui è demandata la misurazione della ricchezza dei sudditi e il prelievo dei ricavi fiscali. I parlamenti sono interpretati come strutture istituzionali atte a facilitare gli scambi tra gli attori, dato il potere di contrattazione relativo ${ }^{26}$. Infine, il modello viene esteso alle democrazie rappresentative moderne, caratterizzate da una pluralità di gruppi di interesse e da assai più elaborati assetti istituzionali volti a facilitare scambi complessi ${ }^{27}$.

Una fondamentale conclusione di North è che diritti di proprietà inefficienti, che hanno cioè come conseguenza stagnazio-

${ }^{25}$ Lo stato viene definito da North come una organizzazione che gode di un vantaggio comparato quanto a risorse di violenza e della capacità di estrarre ricavi fiscali entro un dato territorio. Cfr. North (1981).

${ }^{26}$ La letteratura sulle origini dei parlamenti mostra come queste istituzioni siano sorte come arene in cui i monarchi, costretti dalle pressioni belliche, negoziavano con i sudditi i prelievi fiscali. Cfr. ad es. Zolberg (1980), Bates e Lien (1985) e Levi (1988) e la letteratura di carattere storico ivi citata.

${ }^{27} \mathrm{E}$ interessante notare che North incorpora a questo punto i risultati analitici dei modelli presentati nelle pagine precedenti, integrandoli in un più compiuto framework teorico. 
ne e declino economico, costituiscono la regola piuttosto che l'eccezione. Questo esito è la risultante dell'interazione tra due variabili: la distribuzione del potere di contrattazione e $\mathrm{i}$ costi di transazione, cioè $i$ costi di informazione e $i$ costi di negoziazione e rispetto degli accordi. L'ipotesi è che i governanti tenteranno di stabilire istituzioni che minimizzano $i$ costi di transazione. L'effetto della variabile costi di transazione è tuttavia mediato dal potere di contrattazione relativo: $i$ ricavi fiscali che possono essere ottenuti attraverso una struttura inefficiente di diritti di proprietà, per esempio attraverso la «vendita» di privilegi monopolistici, possono essere maggiori di quelli ottenuti con un sistema che incentiva maggiormente la crescita economica ma che presenta alti costi di informazione e di controllo; oppure i governanti, interessati alla «sopravvivenza politica», possono accettare diritti di proprietà inefficienti piuttosto che alienarsi potenti gruppi di constituents.

La Francia e l'Inghilterra agli inizi dell'epoca moderna forniscono un appropriato terreno per valutare il potenziale esplicativo di queste ipotesi. In Francia il potere di contrattazione quasi assoluto della Corona si combina ad elevati costi di misurazione del reddito e della ricchezza, provenienti da fonti disperse, e ad elevati costi di negoziazione con una molteplicità di assemblee locali. Il declino economico è conseguenza dell'instaurazione di diritti di proprietà inefficienti - la vendita di privilegi monopolistici attraverso l'uso del preesistente sistema delle gilde - e della creazione di un apparato burocratico gigantesco anche se non standardizzato, che comporta elevati costi di agenzia. In Inghilterra l'importanza della tassazione sui beni mobili, legati alle attività commerciali, e la presenza di una fonte primaria di ricavo fiscale (il commercio della lana), aumenta la dipendenza della Corona da potenti gruppi di sudditi, con i quali risulta più vantaggiosa la negoziazione centralizzata dell'entità del prelievo fiscale. La riscossione delle tasse attraverso il Parlamento, che costituisce un forum per transazioni ripetute e un meccanismo di controllo dell'esecuzione degli accordi, abbassa notevolmente $i$ costi di transazione e di agenzia.

Mentre consente di derivare interessanti ipotesi predittive ed esplicative circa la struttura assunta dall'apparato burocratico e dai sistemi fiscali in presenza di differenti combinazioni di variabilii $^{28}$, questa analisi è particolarmente importante nella mi-

${ }^{28}$ Levi (1988) analizza le variazioni nelle politiche fiscali e nella struttura assunta 
sura in cui contribuisce a spiegare nei termini della teoria dell'azione razionale lo sviluppo delle istituzioni rappresentative moderne. Focalizzandosi sul disegno delle istituzioni governative e fiscali introdotte in Inghilterra alla fine del Seicento, dopo che la Gloriosa Rivoluzione metteva fine a una serie di infruttuosi tentativi culminati nella fallita restaurazione della monarchia, North e Weingast (1989) interpretano le innovazioni istituzionali come espliciti tentativi di alterare gli incentivi dei governanti in modo coerente con le preferenze dei vincitori della rivoluzione. In particolare tali mutamenti - che coincidono con la limitazione dei poteri legislativi e giudiziari della Corona e il rafforzamento del ruolo del Parlamento nell'allocazione dei fondi e nel controllo della spesa - riflettono un esplicito tentativo di rendere credibili le promesse della Corona a onorare i propri impegni, prevenendo la possibilità dell'esercizio di un arbitrario potere di confisca. In presenza di una forte controparte parlamentare, la necessità di assicurare la credibilità degli impegni spinge i monarchi alla concessione e alla protezione di diritti vincoli imposti sul proprio comportamento futuro - in cambio di ricavi fiscali. È questa la strada che dal governo «predatorio» conduce alla struttura di garanzie reciproche, o centri multipli di potere di veto, che caratterizza le istituzioni rappresentative. A loro volta, queste innovazioni istituzionali rafforzano la prevedibilità delle decisioni governative $e$, aumentando enormemente la sicurezza dei diritti di proprietà, aprono la strada allo sviluppo economico. Dirigendo l'attenzione sulle istituzioni come strumenti di collective commitment, questa analisi consente di interpretare una parte importante - anche se non esaustiva - dello sviluppo delle istituzioni rappresentative in Inghilterra ${ }^{29}$. In questo modo la prospettiva contrattuale si mostra capace di competere con approcci più tradizionali anche sul difficile terreno dell'analisi storico-comparata, fino a poco tempo fa in larga misura inesplorato con gli strumenti della metodologia dell'attore razionale, senza che il trade-off tra semplificazione anali:

dal prelievo fiscale in base allo schema analitico mutuato da North, a cui aggiunge una terza variabile, il ruolo delle aspettative future che abbassano o elevano il valore della cooperazione nel breve periodo (time discount rates). L'ipotesi - controllata attraverso quattro case-studies - è che il disegno organizzativo dei sistemi fiscali possa venire spiegato e previsto in base alla differente combinazione di tre variabili: potere di contrattazione, costi di transazione e «ombra del futuro».

${ }^{29}$ North e Weingast chiariscono che si tratta di una ricostruzione parziale, che non ignora ma tiene costanti altri fattori rilevanti, quali il ruolo degli attori religiosi. 
tica di processi storici complessi e potere esplicativo si risolva a scapito di quest'ultimo.

\section{Conclusioni}

Questo lavoro non ha inteso proporre una rassegna esaustiva, ma presentare alcuni tra i più importanti sviluppi del programma di ricerca neo-istituzionalista, allo scopo di mostrare la rilevanza del contributo della teoria della scelta razionale all'analisi delle istituzioni politiche.

La convergenza tra analisi spaziale delle istituzioni condotta all'interno della teoria politica positiva e analisi in termini di costi di transazione esprime innanzitutto il potenziale di cumulatività dei risultati analitici perseguiti con l'impiego di una metodologia comune, anche se all'interno di tradizioni di ricerca distinte e con l'impiego di strumenti differenti.

In secondo luogo essa sottolinea come la generalizzazione del paradigma contrattuale all'analisi dello scambio legislativo e delle istituzioni politiche possa contribuire ad un autonomo sviluppo dell'intero approccio. Mentre cade, in questo modo, la pretesa dell' «imperialismo» economico, risulta sempre più obsoleto il punto di vista secondo cui l'impiego della metodologia dell'attore razionale in politica costituirebbe una mera «applicazione», cioè una semplice trasposizione degli strumenti analitici applicati con successo dall'economia. Al contrario, si può affermare che l'analisi comparata delle istituzioni stia delineando un settore di indagine in cui risultano ridefiniti i convenzionali confini disciplinari, gettando le basi di una autentica teoria generale delle istituzioni politiche ed economiche che utilizza una varietà di apporti teorici - qui se ne sono illustrati alcuni - entro l'approccio della scelta razionale.

Se queste implicazioni metodologiche sono per lo più interne all'approccio, sotto il profilo esterno, o del dialogo tra teoria della scelta razionale e scienza politica post-comportamentista, il programma di ricerca neo-istituzionalista presenta caratteristiche tali da sollecitare un confronto aperto. Da questo punto di vista sottolineo almeno due aspetti importanti: l'espansione dell'insieme di strumenti analitici e l'accentuazione dell'orientamento positivo, derivante dal consapevole abbandono di un punto di vista fondazionale che caratterizza tanta parte della riflessione in questo ambito e, conseguentemente, dalla scelta di 
un livello di astrazione inferiore ${ }^{30}$. Sotto questo aspetto, il problema non è tanto quello di ipotizzare la fuoriuscita dallo stato di natura attraverso un modello astratto, quanto piuttosto quello di spiegare l'insorgenza e il funzionamento di assetti istituzionali storicamente dati mediante ipotesi controllabili empiricamente. Come è stato recentemente osservato, «colmare il divario» tra modelli formali e ricerca empirica sembra il tratto comune di un sempre maggior numero di lavori che adottano la prospettiva dell'azione razionale (Ostrom 1991). Mentre queste caratteristiche vanno incontro alle esigenze di realismo più volte sottolineate dai critici dell'approccio, il restare ancorati saldamente alla metodologia dell'attore razionale consente la cumulatività e il rigore della teoria deduttiva.

Dal punto di vista sostantivo, si delinea un terreno straordinariamente promettente per lo sviluppo di una teoria delle istituzioni politiche. Sottolineando due caratteristiche chiave delle istituzioni, come strumenti consapevolmente designati a produrre stabili flussi di decisioni e a risolvere problemi di enforcement dello scambio, questa analisi dirige l'attenzione e offre gli strumenti per trattare aspetti e problemi finora trascurati - costi di informazione e di controllo, credibilità degli impegni e così via - che rendono conto della scelta di assetti istituzionali alternativi.

Non ho finora discusso le obiezioni che sono state mosse a questo tipo di approccio, sia perché questo lavoro aveva come scopo principale dare un'idea delle potenzialità, piuttosto che metterne in rilievo i limiti, sia per il taglio espositivo, molto generale, adottato in questa sede. Tali obiezioni possono essere raggruppate in due insiemi. Il primo, più interno all'approccio formale, scaturisce dall'elaborazione teorica di una famiglia di modelli alternativi ai SIE, centrati sul modo in cui le istituzioni sono designate a risolvere razionalmente problemi di informazione. Secondo questa direzione di ricerca, concentrarsi sulle implicazioni distributive delle istituzioni trascura i vari modi in cui esse consentono di catturare i benefici collettivi provenienti dalla specializzazione. In questa prospettiva il fuoco è sulla scelta di regole e procedure che forniscono agli attori legislativi incentivi a sviluppare policy expertise - cioè competenza relativa

${ }^{30}$ Con questo non si intende affatto negare l'importanza di questa riflessione, alla quale peraltro si deve il pushing the boundaries dell'approccio razionale nel suo complesso e l'individuazione dei suoi limiti. 
alle conseguenze di policies alternative - a basso costo e a condividere tale informazione indipendentemente dal conflitto di interesse sugli esiti del processo decisionale. Benché esistano sensibili differenze tra questi modelli e i SIE dal punto di vista degli assunti comportamentali e delle previsioni empiriche, essi possono essere considerati in una certa misura complementari ai precedenti ${ }^{31}$.

Un secondo insieme di obiezioni è rivolto alla logica generale di questo approccio, e ne tocca immediatamente le ambizioni «endogenizzanti». Secondo queste critiche, efficacemente sintetizzate da Moe (1990), l'analisi neo-istituzionalista può essere accettata solo in quanto ricostruzione esplicativa parziale. Partire dal voto e focalizzarsi sul Congresso, e in generale sulle istituzioni legislative, offusca l'aspetto chiave della «structural choice», cioè la sostanza della politica in quanto lotta per l'esercizio dell'autorità tra attori dotati di potere di contrattazione diseguale. In breve, questo insieme di obiezioni tocca due punti, che possono essere così sintetizzati: a) l'accento sulle istituzioni come strumenti che facilitano la cooperazione trascura l'elemento non volontario tipico dello scambio politico e l'aspetto coercitivo delle istituzioni; b) anziché essere concepite come il prodotto consapevole di scelte razionali allo scopo di minimizzare $\mathrm{i}$ costi di transazione, le istituzioni sono meglio interpretabili come conseguenze non intenzionali di qualche altra motivazione, in particolare come l'esito della lotta per l'esercizio dell'autorità. Questo secondo punto solleva la questione del funzionalismo implicito che sarebbe presente in talune formulazioni, compresa quella di Weingast e Marshall'2.

Benché la portata della questione esiga una riflessione più ampia che esula dagli scopi di questo lavoro, è possibile indicare le linee lungo le quali una risposta potrebbe essere argomentata. Nell'ambito degli studi sul Congresso, se talune «sfumature» funzionaliste - peraltro largamente frutto di ambiguità di linguaggio - sono effettivamente rintracciabili nel modello di Weingast e Marshall, occorre guardare ad altri modelli complementari ad esso. Gamm e Shepsle (1989) hanno analiz-

31 Queste differenze riguardano fondamentalmente le assunzioni cognitive. Cfr. Krehbiel (1990) per un ampio resoconto critico.

32 Nei termini di Moe (1990b, 7), «una questione che resta irrisolta è perché i legislatori e gli altri politici, dato il modo in cui i loro incentivi sono strutturati, dovrebbero preoccuparsi per cominciare dell'efficienza». Per una analoga critica a Williamson ofr. Bowman (1989). 
zato la fase formativa del sistema delle commissioni permanenti nel Congresso, che divenne operante prima alla Camera poi al Senato nel periodo compreso tra il 1810 e il 1825 . Questa ricostruzione storica è condotta avendo in mente due teorie del mutamento istituzionale: la prima vede l'insorgenza e il mutamento delle istituzioni come il prodotto del calcolo razionale degli agenti, il che non significa escludere conseguenze non intenzionali. La seconda sottolinea il grado in cui le istituzioni evolvono in modo non pianificato dagli agenti, come risposta adattiva a shock esogeni. La conclusione degli autori va nella prima direzione, mostrando il ruolo giocato dallo Speaker della Camera Henry Clay nell'innovazione istituzionale. La creazione del sistema delle commissioni permanenti è interpretabile come il prodotto del comportamento razionale di un leader interessato a mantenere il controllo su una coalizione che andava frammentandosi per rafforzare la sua posizione di candidato alla Presidenza. Questo assetto istituzionale ha però mostrato sorprendenti caratteristiche di durata e di efficienza nell'abbassare i costi dello scambio legislativo. Nei termini di Shepsle, «ciò che una istituzione facilita può essere un sottoprodotto di quello che i suoi fondatori intendevano» $(1989 a, 141)$. Più in generale, l'insieme dei lavori di Shepsle fa emergere un resoconto del problema della stabilità e del cambiamento delle istituzioni in cui la scelta degli assetti istituzionali è determinata dalle preferenze per gli esiti ad esse associati e la struttura delle istituzioni politiche riflette i vested interests degli attori coinvolti.

Dal canto loro, North, Weingast e altri hanno messo in luce con sufficiente chiarezza come l'adozione della prospettiva contrattuale per spiegare le istituzioni politiche non abbia come esito quello di ignorarne la specificità. Al contrario, la sfida consiste proprio nel modellare la diversità delle istituzioni economiche e politiche all'interno del medesimo quadro analitico. Come ha mostrato Buchanan, riconoscere che il contesto politico è caratterizzato da elementi coercitivi non preclude la possibilità di analizzare gli assetti politico-istituzionali in termini di scambio. Da una angolazione positiva anziché normativa, North ha evidenziato come gli assetti istituzionali siano interpretabili come l'esito di accordi volti ad abbassare i costi di transazione, o a ridurre l'incertezza dello scambio, dato il potere di contrattazione relativo. In questa prospettiva il tentativo di disegnare istituzioni politiche che minimizzino $i$ costi di transazione è ine- 
stricabilmente connesso alla lotta per il controllo dell'autorità politica, come mostra la ricostruzione dell'evoluzione delle istituzioni rappresentative. Inoltre, la conclusione fondamentale di North - relativa alla frequenza storica di diritti di proprietà inefficienti - colloca la chiave della loro persistenza nelle inefficienze del «mercato politico», ovvero nei tentativi di conservare il potere di contrattazione nell'arena politica (North 1990, 52). Questa conclusione non contrasta con l'assunto della razionalità individuale, se per razionalità si intende l'orientamento alla massimizzazione in presenza di vincoli informativi e strategici, né con la possibilità di valutare comparativamente gli assetti politico-istituzionali in base al criterio dell'efficienza rispetto ai costi di transazione.

In conclusione, le obiezioni di Moe toccano piuttosto il problema del passaggio dai singoli modelli a una teoria più generale che li ricomprenda, e che per ora resta solo in parte adeguatamente specificata, benché il tentativo di North compia un fondamentale passo avanti in questa direzione. Nonostante il fatto che ulteriori sforzi di esplicitazione e di sistematizzazione teorica - sul quale convergono, del resto, i proponenti di questa prospettiva - appaiano necessari, le obiezioni non sembrano pertanto intaccare in profondità il programma di ricerca neoistituzionalista, né offuscarne la portata innovativa.

\section{Riferimenti bibliografici}

Alchian, A. e H. Demsetz (1972), Production, Information Costs and Economic Organization, in «American Economic Review», LXII, pp. 777-795.

Almond, G.A. (1988), The Return to the State, in «American Political Science Review», LXXXII, 3, pp. 853-900.

- (1990), Political Science: A Discipline Divided, Beverly Hills and London, Sage.

Almond, G.A., S.C. Flanagan e R. Mundt (a cura di) (1973), Crisis, Choice and Change, Boston, Little \& Brown.

Akerlof, G. (1970), The Market for «Lemons»: Quality, Uncertainty and Market Mechanisms, in "Quarterly Journal of Economics», LXXXIV, pp. 488-500.

Axelrod, R. (1984), The Evolution of Cooperation, New York, Basic Books; trad. it. Giochi di reciprocità, Milano, Feltrinelli, 1985.

Arrow, K.J. (1963), Social Choice and Individual Values, New York, Wiley, $2^{2}$ edizione; trad. it. Scelte sociali e valori individuali, Milano, Etas Kompass, 1974. 
Barney, J.B. e W.G. Ouchi (1986), Organizational Economics, San Francisco, Jossey Bass.

Bates, R.H. e D. Lien (1985), A Note on Taxation, Development and Representative Government, in «Politics and Society», XIV, pp. 53-70.

Bendor, J. e D. Mookherjee (1987), Institutional Structure and the Logic of Ongoing Collective Action, in «American Political Science Review», LXXXI, pp. 129-54.

Black, D. (1948), On The Rationale of Group Decision-Making, in «Journal of Political Economy», LVI, pp. 23-34.

- (1958), The Theory of Committees and Elections, London, Cambridge University Press.

Bowman, J. (1989), Transaction Costs and Politics, in «Archives européenne de sociologie», XXX, pp. 150-168.

Buchanan, J.M. e G. Tullock (1962), The Calculus of Consent. Logical Foundations of Constitutional Democracy, Ann Arbor, University of Michigan Press.

Coase, R. (1937), The Nature of the Firm, in «Economica», IV, pp. 386-485; trad it. in G. Brosio (a cura di), La teoria economica dell'organizzazione, Bologna, Il Mulino, 1989, pp. 167-193.

Downs, A. (1957), An Economic Theory of Democracy, New York, Harper \& Row, 1957; trad. it. Teoria economica della democrazia, Bologna, Il Mulino, 1988.

Eggertsson, T. (1990), Economic Behavior and Institutions, Cambridge, Cambridge University Press.

Enelow, J.M. e M.J. Hinich (1984), The Spatial Theory of Voting. An Introduction, Cambridge, Cambridge University Press.

- (1990), Advances in Spatial Theory of Voting, Cambridge, Cambridge University Press.

Evans, P.B., D. Rueschemeier e T. Skocpol (1985), Bringing The State Back In, Cambridge, Cambridge University Press.

Farquharson, R. (1969), Theory of Voting, New Haven, Yale University Press.

Frey, B.S. (1984), A New View of Economics: Comparative Analysis of Institutions, in «Economia delle Scelte Pubbliche», II, pp. 3-16.

Fudenberg, D. e E. Maskin (1986), The Folk Theorem in Repeated Games with scounting or with Incomplete Information, in «Econometrica», LIV, pp. 533-54.

Gamm, G. e K.A. Shepsle (1989), Emergence of Legislative Institutions: Standing Committees in the House and the Senate, 18101825, in «Legislative Studies Quarterly», XIV, pp. 39-66.

Harsanyi, J.C. (1977), Rational Behavior and Bargaining Equilibrium in Games and Social Situations, Cambridge, Cambridge University Press; trad. it. Comportamento razionale e equilibrio di contrattazione, Milano, Il Saggiatore, 1985.

Hetcher, M. (1990), On the Inadequacy of Game Theory for the Solu- 
tion of Real-World Collective Action Problems, in K. Cook e M. Levi (a cura di), The Limits of Rationality, Chicago, The University of Chicago Press, pp. 240-249.

Krasner, S.D. (1984), Approaches to the State: Alternative Conceptions and Historical Dynamics, in «Comparative Politics», XVI, 2, pp. 223-246.

Krehbiel, K. (1988), Spatial Models of Legislative Choice, in «Legislative Studies Quarterly», XIII, pp. 259-319.

- (1987), Why Are Congressional Committees Powerful?, in «American Political Science Review», LXXXI, pp. 929-35.

- (1990), Information and Legislative Institutions, University of Michigan Press.

Kreps, D.M. e R. Wilson (1982), Reputation and Imperfect Information, in «Journal of Economic Theory», XXVIII, pp. 253-79.

Laver, M. e N. Schofield (1990), Multiparty Governments: The Politics of Coalition in Europe, Oxford, Oxford University Press.

Levi, M. (1988), Of Rule and Revenue, Berkeley, University of California Press.

Lewis, D. (1962), Convention. A Philosophical Study, Cambridge, Cambridge University Press.

Luce, R.D. e H. Raiffa (1957), Games and Decisions, New York, Wiley.

March, J.G. e J.P. Olsen (1984), The New Institutionalism: Organizational Factors in Political Life, in «American Political Science Review», LXXXVIII, pp. 734-749.

- (1989), Rediscovering Institutions, New York, The Free Press; trad. it. Riscoprire le istituzioni, Bologna, Il Mulino, 1992.

Martelli, P. (1983), La logica della scelta collettiva, Milano, Il Saggiatore.

McKelvey, R.D. (1976), Intransitivities in Multidimensional Voting Models and Some Implications for Agenda Control, in «Journal of Economic Theory», XII, pp. 472-82.

Milgrom, P. e J. Roberts (1988), An Economic Approach to Influence Activities in Organizations, «American Journal of Sociology», XCIV, supplemento speciale, pp. 154-79.

Miller, N.R., B. Grofman e S.L. Feld (1989), The Geometry of Maiority Rule, in «Journal of Theoretical Politics», I, 379-406.

Moe, T.M. (1984), The New Economics of Organization, in «American Journal of Political Science», XXVIII, pp. 739-777.

- (1990a), The Politics of Structural Choice: Toward a Theory of Public Bureaucracy, in O.A. Williamson (a cura di), Organization Theory, Oxford University Press, pp. 116-153.

- (1990b), Political Institutions: The Neglected Side of the Story, in «Journal of Law, Economics and Organization», VI.

Monroe, K.R. (a cura di) (1990) The Nature of Contemporary Political 
Science: A Roundtable Discussion, in «PS: Political Science \& Politics», XXIII, 1, pp. 34-44.

- (1991), The Economic Approach to Politics, New York, Harper Collins.

Morlino, L. (1989), Epitaffio per un approccio di successo: il sistema politico, in A. Panebianco (a cura di), L'analisi della politica. Tradizioni di ricerca, modelli, teorie, Bologna, Il Mulino, pp. 71-87.

Mueller, D.C. (1989), Public Choice II, Cambridge, Cambridge University Press.

Nordlinger, E.A. (1987), Taking the State Seriously, in S.P. Huntington e J.M. Nelson, Understanding Political Development, Beverly Hills and London, Sage, pp. 353-388.

North, D.C. (1990), Institutions, Institutional Change and Economic Performance, Cambridge, Cambridge University Press.

- (1981), Structure and Change in Economic History, New York, Norton.

North, D.C. e B.R. Weingast (1989), Constitutions and Commitment: Evolutions of the Institutions Governing Public Choice in 17th Century England, in «Journal of Economic History», II, pp. 80332.

Ordeshook, P.C. (1990), The Emerging Discipline of Political Economy, in J.E. Alt e K.A. Shepsle (a cura di), Perspectives on Positive Political Theory, Cambridge, Cambridge University Press.

Ordeshook, P.C. e K.A. Shepsle (a cura di) (1981), Political Equilibrium, Boston, Kluwer-Nijhoff.

Ostrom, E. (1991), Rational Choice Theory and Institutional Analysis: Toward Complementarity, in «American Political Science Review», LXXXV, pp. 237-43.

Plott, C.R. (1967), A Notion of Equilibrium and Its Possibility Under Maiority Rule, in «American Economic Review», LVII, pp. 787-806.

- (1976), Axiomatic Social Choice Theory: An Overview and Interpretation, in «American Journal of Political Science», pp. 511-95.

Rawls, J. (1971), A Theory of Justice, Cambridge, Cambridge University Press; trad. it. Una teoria della giustizia, Milano, Feltrinelli, 1982.

Richter, R. (1984), The New Institutional Economics, in «Journal of Institutional and Theoretical Economics», CXI, pp. 1-6.

Riker, W.H. (1962), The Theory of Political Coalitions, New Haven, Yale University Press.

- (1980), Implications From the Disequilibrium of Maiority Rule for the Study of Institutions, in «American Political Science Review», LXXIV, pp. 432-446.

- (1990), Political Science and Rational Choice, dattiloscritto, Università di Rochester.

Schelling, T. (1960), The Strategy of Conflict, Cambridge, Harvard University Press, $2^{2}$ ed. 1980. 
Schofield, N. (1978), Instability of Simple Dynamic Games, in «The Review of Economic Studies», XLV, pp. 575-94.

Schotter, A. (1981), The Economic Theory of Social Institutions, Cambridge, Cambridge University Press.

Shepsle, K.A. (1979), Institutional Arrangements and Equilibrium in Multidimensional Voting Models, in «American Journal of Political Science», XXIII, pp. 27-59.

- (1986), Institutional Equilibrium and Equilibrium Institutions, in H. Weisberg (a cura di), Political Science: The Science of Politics, New York, Agathon Press, pp. 51-81.

- (1989), Studying Institutions: Some Lessons From the Rational Choice Approach, in «Journal of Theoretical Politics», I, pp. 131147.

Shepsle, K.A. e M. Laver (1990a), Coalitions and Cabinet Government, in «American Political Science Review», LXXXIV, 3, pp. 873-891.

- (1990b), Government Coalitions and Intraparty Politics, in «British Journal of Political Science», XX, pp. 489-507.

- (1990c), Size Principle and Minority Cabinets, paper presentato alla Conferenza in onore di W.H. Riker, Rochester, 12-13 ottobre.

Shepsle, K.A. e B.R. Weingast (1981), Structure-Induced Equilibrium and Legislative Choice, in «Public Choice», XXXVII, pp. 221-37.

- (1987), Foundations of Committee Power, in «American Political Science Review», LXXXI, pp. 85-104.

Simon, H.A. (1957), Models of Man, New York, Wiley.

Strom, K. (1990), Minority Governments and Maiority Rule, Cambridge, Cambridge University Press.

Taylor, M. (1976), Anarchy and Cooperation, New York, Wiley.

Tirole, J. (1986), Hierarchies and Bureaucracies, in «Journal of Law, Economics and Organization», II, pp. 235-59.

Tullock, G. (1981), Why So Much Stability?, in «Public Choice», XXXVII, pp. 189-202.

Ulmann-Margalit, E. (1977), The Emergence of Norms, Cambridge, Cambridge University Press.

Urbani, G. (1989), La teoria del sistema politico: prospettive, in A. Panebianco (a cura di), L'analisi della politica. Tradizioni di ricerca, modelli, teorie, Bologna, Il Mulino, pp. 89-102.

Weingast, B.R. (1990), The Role of Credible Commitments in State Finance, in «Public Choice», LXVI, pp. 89-97.

Weingast, B.R. e W. Marshall (1988), The Industrial Organization of Congress: or Why Legislatures, Like Firms, Are Not Organized as Markets, in «Journal of Political Economy», XCVI, pp. 132-163; trad. it. parziale in G. Brosio (a cura di), La teoria economica dell'organizzazione, Bologna, ll Mulino, 1989, pp. 435-467.

Williamson, O.A. (1975), Markets and Hierarchies, New York, The Free Press. 
- (1985), The Economic Institutions of Capitalism: Firms, Markets, Relational Contracting, New York, The Free Press; trad. it. Le istituzioni economiche del capitalismo, Milano, Franco Angeli, 1988.

- (1990a), Organization Theory. From Chester Barnard to Now, Oxford, Oxford University Press.

- (1990b), A Comparison of Alternative Approaches to Economic Organization, in «Journal of Institutional and Theoretical Economics», CXIVI, pp. 61-71.

Zolberg, A.R., Strategic Interaction and the Formation of Modern States: France and England, in «International Social Science Journal», XXXII, 687-716. 